\title{
Correlation studies for B-spline modeled F2 Chapman parameters obtained from FORMOSAT-3/COSMIC data
}

\author{
M. Limberger ${ }^{1,2}$, W. Liang ${ }^{2}$, M. Schmidt ${ }^{2}$, D. Dettmering ${ }^{2}$, M. Hernández-Pajares ${ }^{3}$, and U. Hugentobler ${ }^{1}$ \\ ${ }^{1}$ Technische Universität München - Institute of Astronomical and Physical Geodesy (IAPG), Arcisstr. 21, \\ 80333 Munich, Germany \\ ${ }^{2}$ Deutsches Geodätisches Forschungsinstitut (DGFI), Alfons-Goppel-Str. 11, 80539 Munich, Germany \\ ${ }^{3}$ UPC-IonSAT Research Group, Universitat Politècnica de Catalunya (UPC), Barcelona, Spain
}

Correspondence to: M. Limberger (marco.limberger@dgfi.badw.de)

Received: 9 June 2014 - Revised: 11 November 2014 - Accepted: 19 November 2014 - Published: 17 December 2014

\begin{abstract}
The determination of ionospheric key quantities such as the maximum electron density of the F2 layer $N m \mathrm{~F} 2$, the corresponding $\mathrm{F} 2$ peak height $h m \mathrm{~F} 2$ and the $\mathrm{F} 2$ scale height $H \mathrm{~F} 2$ are of high relevance in 4-D ionosphere modeling to provide information on the vertical structure of the electron density $\left(N_{\mathrm{e}}\right)$. The $N_{\mathrm{e}}$ distribution with respect to height can, for instance, be modeled by the commonly accepted F2 Chapman layer. An adequate and observation driven description of the vertical $N_{\mathrm{e}}$ variation can be obtained from electron density profiles (EDPs) derived by ionospheric radio occultation measurements between GPS and low Earth orbiter (LEO) satellites. For these purposes, the six FORMOSAT-3/COSMIC (F3/C) satellites provide an excellent opportunity to collect EDPs that cover most of the ionospheric region, in particular the F2 layer. For the contents of this paper, F3/C EDPs have been exploited to determine $N m \mathrm{~F} 2, h m \mathrm{~F} 2$ and $H \mathrm{~F} 2$ within a regional modeling approach. As mathematical base functions, endpointinterpolating polynomial B-splines are considered to model the key parameters with respect to longitude, latitude and time. The description of deterministic processes and the verification of this modeling approach have been published previously in Limberger et al. (2013), whereas this paper should be considered as an extension dealing with related correlation studies, a topic to which less attention has been paid in the literature. Relations between the B-spline series coefficients regarding specific key parameters as well as dependencies between the three F2 Chapman key parameters are in the main focus. Dependencies are interpreted from the postderived correlation matrices as a result of (1) a simulated scenario without data gaps by taking dense, homogenously dis-
\end{abstract}

tributed profiles into account and (2) two real data scenarios on 1 July 2008 and 1 July 2012 including sparsely, inhomogeneously distributed F3/C EDPs. Moderate correlations between $h m \mathrm{~F} 2$ and $H \mathrm{~F} 2$ as well as inverse correlations between $N m \mathrm{~F} 2$ and $H \mathrm{~F} 2$ are reflected from the simulation. By means of the real data studies, it becomes obvious that the sparse measurement distribution leads to an increased weighting of the prior information and suppresses the parameter correlations which play an important role regarding the parameter estimability. The currently implemented stochastic model is in need of improvement and does not consider stochastic correlations which consequently cannot occur.

Keywords. Ionosphere (equatorial ionosphere; modeling and forecasting) - radio science (ionospheric physics)

\section{Introduction}

One of the major tasks in ionospheric research activities concerns the determination of physically relevant parameters from space geodetic observations. Exploiting the knowledge about the physical processes improves the description of the ionospheric behavior in time and space to monitor ionosphere phenomena and perform space weather studies. One of the most important parameters in this context is the electron density $\left(N_{\mathrm{e}}\right)$ distribution, which constitutes the focus in several current ionospheric modeling approaches and enables the study of ionospheric storms, ion composition and effects on radio communications between satellites and ground receivers. The ability to describe $N_{\mathrm{e}}$ variations in space and time is an important step along the way of understanding still 
unsolved ionospheric phenomena affected by Sun-Earth interactions and to improve existing ionosphere models.

Various global (e.g., Hernández-Pajares et al., 1999; Azpilicueta et al., 2006; Todorova et al., 2007) and regional ionosphere models (e.g., Dettmering et al., 2011) describe the spatiotemporal distribution of the vertical total electron content (VTEC). Beside novelties in the application of different mathematical and physical modeling approaches, several modeling strategies nowadays are based on the combination of different measurement techniques to compensate for the diversity in the data resolution and to achieve the best possible measurement distribution. Terrestrial permanent networks, e.g., the International GNSS Service (IGS) tracking stations (Dow et al., 2009; Hernández-Pajares, 2004; Hernández-Pajares et al., 2009) and the Sistema de Referencia Geocéntrico para las Américas (SIRGAS) (Sánchez et al., 2013), have been deployed for the tracking of global navigation satellite systems (GNSS) and provide slant total electron content (STEC) data with a high spatial and temporal resolution over the continents.

Complementary, VTEC over the oceans can be derived from radar altimetry (RA) along the satellite orbits. On most of the current RA missions such as Jason-2, SARAL, Cyosat and HY-2A, a DORIS (Doppler Orbitography and Radiopositioning Integrated by Satellite) receiver that is active for orbit determination purposes can also be considered to derive STEC from the signals transmitted by DORIS ground beacons (Dettmering et al., 2014). The total electron content (TEC) is commonly considered as the backbone of ionosphere models, but its integral characteristic (TEC is defined as the integral of the $N_{\mathrm{e}}$ along any specific signal path) makes the TEC observable insensitive for the description of the $N_{\mathrm{e}}$ distribution, especially with respect to height.

However, empirical models such as NeQuick (Nava et al., 2008) and the International Reference Ionosphere (IRI) (Bilitza et al., 2011) provide global 4-D $N_{\mathrm{e}}$ descriptions, mainly driven by ionosonde parameters such as the layerdependent critical frequencies $f_{0} \mathrm{E}$ ( $\mathrm{E}$ layer), $f_{0} \mathrm{~F} 1$ ( $\mathrm{F} 1$ layer), $f_{0} \mathrm{~F} 2$ (F2 layer) or the maximum usable frequency factor $\mathrm{M}(3000) \mathrm{F} 2$ (ratio of maximum usable frequency at a distance of $3000 \mathrm{~km}$ and $f_{0} \mathrm{~F} 2$ ). In addition to the provision of TEC data, the La Plata Ionospheric Model (LPIM) (Azpilicueta et al., 2006) incorporates space-based STEC measurements as tracked by satellites in low Earth orbit (LEO) to retrieve electron density information. In addition, several assimilation schemes of different complexity and relying on different kinds of data have been developed - for instance, GAIM (Schunk et al., 2001) and EDAM (Angling, 2008).

In order to obtain information of the $N_{\mathrm{e}}$ distribution from satellite measurements, ionospheric GPS radio occultations (IRO) (see, for example, Kirchengast et al. (2004) or Jakowski et al. (2004)), can be considered as an adequate observation type with height sensitivity. Relevant information of the $N_{\mathrm{e}}$ can be derived from GPS signal delay changes during an occultation event caused by atmospheric influences.
IRO can be used to derive electron density profiles (EDPs) and therefore allow for modeling of the $N_{\mathrm{e}}$ distribution with physically relevant key parameters with height dependence. For instance, the $\mathrm{F} 2$ peak height $h m \mathrm{~F} 2$ and $\mathrm{F} 2$ scale height $H F 2$ play an essential role in this context and contribute to the definition of the vertical $N_{\mathrm{e}}$ profile shape. Various model approaches have already been proposed, and recently published papers will be mentioned here: Altadill et al. (2012) modeled $h m \mathrm{~F} 2$ from globally distributed ionosonde measurements by means of a spherical harmonic analytical model for quiet solar conditions, Hoque and Jakowski (2012) proposed the operational Neustrelitz peak height model based on 13 coefficients with only a few empirically fixed parameters and driven by ionosonde data plus IRO measurements, and Brunini et al. (2013) studied and compared different techniques to estimate $h m F 2$. Moreover, Alizadeh (2013) used spherical harmonic expansions to model the $N_{\mathrm{e}}$ distribution globally from the combination of different satellite-based observation techniques and Limberger et al. (2013) dealt with the estimation of the three F2 Chapman parameters $N m \mathrm{~F} 2$, $h m \mathrm{~F} 2$ and $H \mathrm{~F} 2$ in terms of a polynomial B-spline representation from IRO-derived electron density profiles (EDPs), which was further extended by Liang et al. (2014) for the usage of EDPs together with ground-based GPS data.

It stands to reason that, depending on the choice of key parameters, the question of parameter interdependencies arises and will form the emphasis of the investigations in this paper. Several studies dealing with the issue of correlations between ionospheric parameters are purely based on ionosonde measurements. To mention just a few of these, Zhang et al. (2006) and also Liu et al. (2006) studied correlations between the ionogram-derived Chapman scale height $H_{T}$ (or $\mathrm{Hm}$ ) and the $\mathrm{F} 2$ peak parameters $f_{0} \mathrm{~F} 2$ and $h m \mathrm{~F} 2$, the bottomside F2 layer thickness parameter $B 0$, and the slab thickness $\tau$; Lee and Reinisch (2007) investigated scale height variations from Jicamarca ionograms during quiet solar conditions and thereby analyzed appearing correlations with $f_{0} \mathrm{~F} 2$, $h m \mathrm{~F} 2$ and $B 0$. Instead of using ionosonde measurements, a data basis consisting of EDPs observed by the LEO mission Formosa Satellite 3 and Constellation Observing System for Meteorology, Ionosphere, and Climate (FORMOSAT3/COSMIC, henceforth F3/C) (Rocken et al., 2000) has been established here. Investigations of parameter interdependencies on the regional scale are carried out for three F2 Chapman key parameters which are of fundamental importance in ionospheric research: the maximum electron density $\mathrm{NmF} 2$ of the F2 layer, the F2 peak height $h m \mathrm{~F} 2$ and the F2 scale height $H \mathrm{~F} 2$. These $\mathrm{F} 2 \mathrm{Chapman}$ parameters are required for the description of the F2 layer, which is, at least in large part, contained in the F3/C profiles - the six F3/C satellites fly at orbit heights of approximately $800 \mathrm{~km}$. The modeling fundamentals leading to the determination of these key parameters have already been described in Limberger et al. (2013) and the intention of the subsequent investigations are supplementary with emphasis on correlation studies. 
The basic principles of the modeling concept and parameter determination will therefore be repeated at the beginning of Sect. 2. Starting with a preliminary adjustment model (Sect. 3) that is applied to a simulation scenario (Sect. 4), first studies based on a dense, homogeneous measurement distribution without data gaps are performed. According to the subsequent real data scenario (Sect. 5), the adjustment system is extended (Sect. 6) and will be applied for studies during quiet solar conditions on 1 July 2008 (Sect. 7) and increased solar activity on 1 July 2012 (Sect. 8). Finally, some final conclusions will be given in Sect. 9.

\section{Modeling the vertical $N_{\mathrm{e}}$ distribution}

The description of the vertical $N_{\mathrm{e}}$ distribution follows from an adapted $\alpha$-Chapman function proposed by Jakowski (2005) and considers a F2 Chapman layer with an additional plasmasphere expansion given by

$$
\begin{aligned}
N_{\mathrm{e}}(h) & =N m \mathrm{~F} 2 \exp [0.5(1-z-\exp (-z))] \\
& +N 0 \mathrm{P} \exp (-|h-h m \mathrm{~F} 2| / H \mathrm{P}) \\
& \text { with } z=(h-h m \mathrm{~F} 2) / H \mathrm{~F} 2 .
\end{aligned}
$$

Model parameters are the maximum electron density $N m \mathrm{~F} 2$ of the ionospheric F2 layer, the corresponding F2 peak height $h m \mathrm{~F} 2$ and a constant $\mathrm{F} 2$ topside scale height $H \mathrm{~F} 2$, which constitute the key quantities for this study. The simplifying assumption in considering a constant scale height has been proposed by Reinisch at al. (2004) and, for instance, considered in Stankov and Jakowski (2005) as plasma scale height in the topside ionosphere. The description of the plasmasphere follows from the basis density NOP and plasmasphere scale height $H \mathrm{P}$, where proportionality between $N m \mathrm{~F} 2$ and NOP has been taken into account and $H \mathrm{P}$ is fixed at $H \mathrm{P}=10^{4} \mathrm{~km}(h \geq h m \mathrm{~F} 2)$ and $H \mathrm{P}=10 \mathrm{~km}(h<h m \mathrm{~F} 2)$, respectively, to allow for a smooth transition between the F2and plasmasphere layer.

The model approach presented here is in particular suited for regional applications since localizing endpointinterpolating polynomial B-splines (Lyche and Schumaker, 2000; Schmidt, 2007) are exploited to model the target quantities. $N m \mathrm{~F} 2, h m \mathrm{~F} 2$ and $H \mathrm{~F} 2$ are expressed as tensor products of three 1-D polynomial B-spline functions related to longitude $\lambda$, latitude $\varphi$ and time $t$ with unknown series coefficients. The formulation in terms of B-splines yields

$h m \mathrm{~F} 2(\lambda, \varphi, t)=$

$$
\sum_{k_{1}=1}^{K\left(J_{\lambda}\right)} \sum_{k_{2}=1}^{K\left(J_{\varphi}\right)} \sum_{k_{3}=1}^{K\left(J_{t}\right)} d_{k_{1}, k_{2}, k_{3}}^{J_{\lambda}, J_{\varphi}, J_{t}} \phi_{k_{1}}^{J_{\lambda}}(\lambda) \phi_{k_{2}}^{J_{\varphi}}(\varphi) \phi_{k_{3}}^{J_{t}}(t)
$$

for $h m \mathrm{~F} 2$ and can be accordingly obtained for $N m \mathrm{~F} 2$ and $H \mathrm{~F} 2$. The validity of $\mathrm{B}$-splines for ionospheric VTEC modeling, in particular when dealing with inhomogeneously distributed observations, has been successfully demonstrated in
Schmidt et al. (2011) through comparison with spherical harmonics series expansions. Moreover, an exemplary approach for regional VTEC modeling has been published by Dettmering et al. (2011) and later enhanced by Limberger et al. (2013) and Liang et al. (2014) to determine F2 Chapman key parameters, based on Eq. (1), aiming for regional 4-D $N_{\mathrm{e}}$ representation.

Thus, the target quantities to be determined are the Bspline series coefficients $d_{k_{1}, k_{2}, k_{3}}^{J_{\lambda}, J_{\varphi}, J_{t}}$ depending on the B-spline levels $J_{\lambda}, J_{\varphi}$ and $J_{t}$, which are typically adapted to the average measurement density and finally define the degree of smoothing and model resolution. The number $K(J)$ of coefficients related to $\lambda, \varphi$ and $t$ can be calculated from $K=$ $2^{J}+2$ and the total number of unknowns (for a specific key parameter) accordingly results from $N_{u}=2^{J_{\lambda}}+2^{J_{\varphi}}+2^{J_{t}}+6$. In general, all series coefficients are collected in the vector of unknowns $\boldsymbol{d}=\left[\boldsymbol{d}_{N m \mathrm{~F} 2}^{T}, \boldsymbol{d}_{h m \mathrm{~F} 2}^{T}, \boldsymbol{d}_{H \mathrm{~F} 2}^{T}\right]^{T}$ as defined in a Gauss-Markov model; it is important to note here that, due to the nonlinearity of Eq. (1), a linearization is required and no absolute coefficients apart from corrections $\Delta \widehat{\boldsymbol{d}}$ for initial series coefficients $\boldsymbol{d}_{0}$ have to be estimated.

\section{Preliminary adjustment system}

Taking the linearized model into account, the estimated set of coefficients results from an iterative estimation procedure via

$\widehat{\boldsymbol{d}}=\boldsymbol{d}_{0}+\Delta \widehat{\boldsymbol{d}}=\boldsymbol{d}_{0}+\mathbf{N}^{-1} \boldsymbol{A}^{T} \mathbf{P} \boldsymbol{L}$,

with $\mathbf{N}=\boldsymbol{A}^{T} \mathbf{P} \boldsymbol{A}$

and includes the design matrix $\boldsymbol{A}$, positive definite observation weight matrix $\mathbf{P}$ and reduced observation vector $\boldsymbol{L}$ containing the differences between observed $N_{\mathrm{e}, \mathrm{F} 3 / \mathrm{C}}$ and initial electron densities $N_{\mathrm{e}}\left(\boldsymbol{d}_{0}\right) . \boldsymbol{d}_{0}$ contains initial values for the series coefficients and is adapted after each iteration step "it" by the outcome of the previous iteration it -1 . Here, the initial coefficients $\boldsymbol{d}_{0}$ are derived in a pre-processing step from the International Reference Ionosphere 2007 (IRI07) (Bilitza, 2000; Bilitza et al., 2011). Values for $N m \mathrm{~F} 2$ as well as $h m \mathrm{~F} 2$ are directly accessible and, after Wright (1960) and Davies (1990), enable the derivation of $H \mathrm{~F} 2=\tau / 4.13$ by taking the slab thickness $\tau=\mathrm{VTEC} / N m \mathrm{~F} 2$ into account (Jayachandran et al., 2004). Based on the initial IRI07 data set, a homogenous data grid can be established, allowing for the determination of initial coefficients $\boldsymbol{d}_{0}$.

Concerning the parameter estimability, Limberger et al. (2013) showed that the presented modeling approach allows for a simultaneous determination of B-spline series coefficients regarding the three related F2 Chapman key parameters $N m \mathrm{~F} 2, h m \mathrm{~F} 2$ and $H \mathrm{~F} 2$ by taking advantage of the sensitivity of EDPs.

In this paper, correlations between the B-spline series coefficients and also F2 Chapman key parameters are studied 
in detail. A reliable separability of the key parameters is certainly required for obtaining realistic values and to ensure a safe convergency of the procedure. To discuss this question, the correlations between key parameters shall be analyzed on the basis of the correlation matrices $\mathbf{K}_{x x}$ and $\mathbf{K}_{t t}$ for coefficients and target key parameters, respectively, where the latter one results from the application of the error propagation law (EPL). The subscripts $x x$ and $t t$ will henceforth be used to identify the association of the matrices to the original unknowns, i.e., the B-spline series coefficients or target key parameters, respectively.

The covariance matrix of the unknown series coefficients $\Sigma_{x x}=\mathbf{N}^{-1}$ is the inverse of the normal equation matrix. The diagonal elements of $\Sigma_{x x}$ are the variances $\widehat{\sigma}_{d_{j}}^{2}$ for $j \in\left\{1, \ldots, N_{u}\right\}$ of the series coefficients $d_{j}$. For the vectorvalued function $\boldsymbol{f}=\mathbf{F} \widehat{\boldsymbol{d}}$, which is representing a set of key parameters as linear functions of the estimated B-spline series coefficients, the EPL can be applied to derive the corresponding covariance matrix $\Sigma_{t t}$ of the key parameters as $\Sigma_{t t}=\mathbf{F} \Sigma_{x x} \mathbf{F}^{T}$. The transformation matrix $\mathbf{F}$ consists of partial derivatives of the key parameters with respect to the series coefficients resulting from Eq. (2). This means particularly that $\mathbf{F}$ contains the spline tensor products - for instance $\partial h m \mathrm{~F} 2 / \partial d_{k_{1}, k_{2}, k_{3}}^{J_{\lambda}, J_{\varphi}, J_{t}}=\phi_{k_{1}}^{J_{\lambda}}(\lambda) \phi_{k_{2}}^{J_{\varphi}}(\varphi) \phi_{k_{3}}^{J_{t}}(t)$ in the case of $h m \mathrm{~F} 2$. The correlation matrices $\mathbf{K}_{x x}$ and $\mathbf{K}_{t t}$ with $\mathbf{K}_{x x_{\mathrm{r}, \mathrm{c}}}=\Sigma_{x x_{\mathrm{r}, \mathrm{c}}} / \sigma_{r} \sigma_{c}$ and $\mathbf{K}_{t t_{\mathrm{r}, \mathrm{c}}}=\Sigma_{t t_{\mathrm{r}, \mathrm{c}}} / \sigma_{r} \sigma_{c}$ are defined as normalized covariance matrices, where $\mathrm{r}$ and $\mathrm{c}$ indicate the row and column indices here.

\section{Simulation}

A stable estimation of B-spline series coefficients applying the adjustment model presented in Eq. (3) requires a dense measurement distribution, i.e., a scenario without data gaps. This will not be the case for a real distribution of $N_{\mathrm{e}}$ profiles in the foreseeable future, but can be simulated to study the parameter correlations. Therefore, a $24 \mathrm{~h}$ timespan, 1 July 2008 , and a region of $\left[250^{\circ}, 340^{\circ}\right]$ longitude and $\left[-60^{\circ}, 30^{\circ}\right]$ latitude (South America) have been selected. Homogeneously distributed EDPs with a resolution of $10^{\circ}(\lambda) \times 10^{\circ}(\varphi) \times 2 \mathrm{~h}$ are simulated, which is dense enough for the selected B-spline level of $J_{\lambda}=2$, $J_{\varphi}=2$ and $J_{t}=3$ to allow for the estimation of all coefficients without any prior information required to bridge data gaps. According to these levels, the total number of unknown coefficients amounts here to 360 per key parameter and 1080 in total. Hence, the dimension of $\mathbf{K}_{x x}$ is $1080 \times 1080$. For the subsequent investigations, $\mathbf{F}$ has been computed for fixed epochs and a geographical resolution of $5^{\circ} \times 5^{\circ}$ (see Sect. 3), leading to a matrix size of $1083 \times 1080$. Consequently, $\mathbf{K}_{t t}$ has a dimension of 3 (key parameters) $\times\left[90^{\circ} / 5^{\circ}+1\right](\varphi) \times\left[90^{\circ} / 5^{\circ}+1\right](\lambda)$ resulting in $1083 \times 1083$ elements for a specific epoch including all three key parameters.

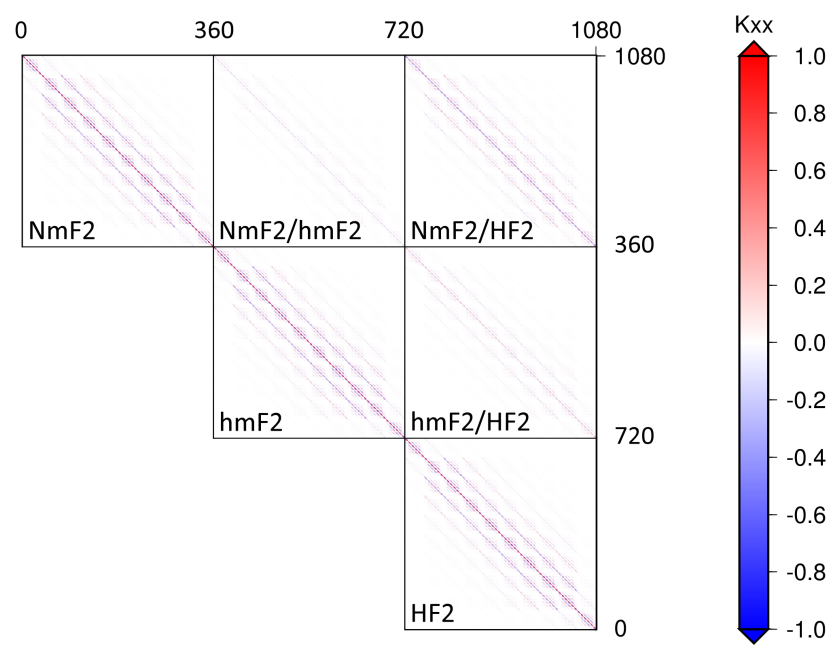

Figure 1. Correlation matrix of the coefficients $\mathbf{K}_{x x}$ for the simulated scenario.

Each simulated profile contains synthetic $N_{\mathrm{e}}$ observations between 100 and $800 \mathrm{~km}$ height with an altitude sampling interval of $10 \mathrm{~km}$. Each synthetic $N_{\mathrm{e}}$ observation is determined by means of Eq. (1), where the $N m \mathrm{~F} 2, h m \mathrm{~F} 2$ and also $H \mathrm{~F} 2$ parameter values are computed from the initial coefficients $\boldsymbol{d}_{0, N m \mathrm{~F} 2}, \boldsymbol{d}_{0, h m \mathrm{~F} 2}$ and $\boldsymbol{d}_{0, H \mathrm{~F} 2}$. For each of these key parameter vectors, an artificial systematic bias has been introduced that is contained in the simulated observations. Since IRI07 has been used to derive $\boldsymbol{d}_{0}$, these offsets can be interpreted as corrections with respect to IRI07 that are reflected in the observations. The systematic biases are then retrieved within a closed-loop procedure. Two percent of the mean $N_{\mathrm{e}}$ per profile is considered to be the standard deviation to simulate a measurement error with zero mean. Thereby, the noise level is adapted to the current solar activity condition. The correlation matrices of the simulated scenario with respect to the coefficients and key parameters are depicted in Figs. 1 and 2.

$\mathbf{K}_{x x}$ contains correlations between all coefficients (i.e., for all key parameters, geographical positions and epochs), where block submatrices are confined to black boxes and labeled with regard to whether referring to $N m \mathrm{~F} 2, h m \mathrm{~F} 2, H \mathrm{~F} 2$ or an off-diagonal submatrix with interparameter-coefficient correlations. All coefficients are ordered by longitude (in the innermost loop) followed by latitude (in the second loop) and time (in the outer loop). The sorting scheme will be explained later in a separate subsection dealing with the matrix composition (see Sect. 7). Correlations are hardly visible in Fig. 1 along the diagonal, most probably related to B-spline dependencies, in particular between the $N m \mathrm{~F} 2$ and $H \mathrm{~F} 2$ coefficients. Although visible in both plots, the correlation structure is clearly enhanced for $\mathbf{K}_{t t}$ in Fig. 2. The small blue off-diagonal patches within each key parameter block indicate a negative correlation of the scaling coefficients for neighboring B-splines, i.e., the increase of a series coefficient value for a specific spline leads to the de- 


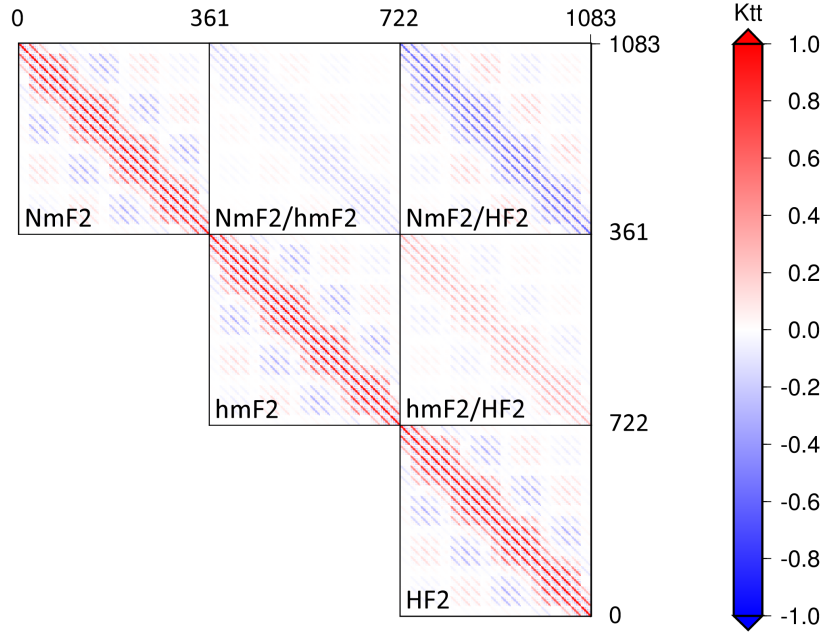

Figure 2. Correlation matrices of the key parameters $\mathbf{K}_{t t}$ for the fixed time moment 12:00 UT of the simulated scenario. $\mathbf{K}_{t t}$ represents correlations for parameters related to a grid with $\Delta \varphi=5^{\circ} \times \Delta \lambda=5^{\circ}$ resolution

crease of another correlated coefficient. This can be anticipated since one key parameter is always defined by three overlapping splines. Furthermore, the colors indicate negative correlations between $N m \mathrm{~F} 2$ and $H \mathrm{~F} 2$ and, although slightly weaker, between $\mathrm{NmF} 2$ and $h m \mathrm{~F} 2$. Moderate positive correlations become visible between $h m \mathrm{~F} 2$ and $H \mathrm{~F} 2$. Nevertheless, the correlations of nearby coefficients and parameters are the most prominent ones. The corresponding numerical values are given in Table 1 for the key parameters. Since the coefficient correlations are considerably weaker, a corresponding table for $\mathbf{K}_{x x}$ is not shown here.

In the first row, Table 1 provides the total minimum and maximum value of $\boldsymbol{K}_{t t}$, followed by detailed information. Below, minimum and maximum values for the submatrices related to the specific key parameters are shown. Further, the correlations within the submatrices are binned into the intervals $[-1,-0.7],]-0.7,-0.3],]-0.3,0[,[0,0.3[,[0.3,0.7[$ and $[0.7,1]$ and are given as a percentage of the number of elements in the corresponding submatrix, excluding the matrix diagonals. The large percentages of correlations are shown in bold. Some cells in Table 1 include underlined numbers to highlight increased correlations beyond the ] $-0.3,0.3$ [ interval.

As already claimed, those correlations originating from Bsplines (appearing close to the diagonal of the $\mathbf{K}_{x x}$ and $\mathbf{K}_{t t}$ matrices) are particularly apparent as characteristic positive and negative dependencies due to the B-spline nature. Furthermore, the height-dependent parameters $h m \mathrm{~F} 2$ and $H \mathrm{~F} 2$ exhibit moderate positive correlations with a maximum of 0.63. $4.6 \%$ of the correlations between $h m \mathrm{~F} 2$ and $H \mathrm{~F} 2$ are located in the range of $[0.3,0.7[$.

This output is based on a simulated scenario and purely shows the correlations between model parameters that are
Table 1. Key parameter correlations contained in $\mathbf{K}_{t t}$ for the simulated scenario for 1 July 2008. Minimum and maximum correlations of the whole matrix as well as for the key parameter-affiliated submatrices are depicted in the specific headlines (grey background). Percentage values related to different correlation intervals are listed beneath, where the dominant correlations are indicated by bold numbers. Increased correlations beyond the ] - 0.3, 0.3[ sector are underlined.

\begin{tabular}{|c|c|c|c|c|c|}
\hline Total $\mathbf{K}_{t t}$ & & Min & -0.62 & Max & 0.98 \\
\hline$N m \mathrm{~F} 2$ & & Min & -0.33 & Max & 0.97 \\
\hline$[-1,-0.7]$ & ]$-0.7,-0.3]$ & ]$-0.3,0[$ & ]0, 0.3[ & {$[0.3,0.7[$} & {$[0.7,1]$} \\
\hline $0.0 \%$ & $0.2 \%$ & $47.8 \%$ & $44.8 \%$ & $\underline{4.6} \%$ & $2.6 \%$ \\
\hline$h m \mathrm{~F} 2$ & & Min & -0.34 & $\operatorname{Max}$ & 0.98 \\
\hline$[-1,-0.7]$ & ]$-0.7,-0.3]$ & ]$-0.3,0[$ & ] $0,0.3[$ & {$[0.3,0.7[$} & {$[0.7,1]$} \\
\hline $0.0 \%$ & $0.3 \%$ & $47.2 \%$ & $45.5 \%$ & $\underline{4.3} \%$ & $2.7 \%$ \\
\hline$H F 2$ & & Min & -0.34 & Max & 0.98 \\
\hline$[-1,-0.7]$ & ]$-0,7,-0.3]$ & ]$-0,3,0[$ & ]0, 0.3[ & {$[0.3,0.7[$} & {$[0.7,1]$} \\
\hline $0.0 \%$ & $0.2 \%$ & $47.3 \%$ & $45.5 \%$ & $\underline{4.3} \%$ & $2.7 \%$ \\
\hline$N m \mathrm{~F} 2, h m \mathrm{~F} 2$ & & Min & -0.31 & $\operatorname{Max}$ & 0.11 \\
\hline$[-1,-0.7]$ & ]$-0.7,-0.3]$ & ]$-0.3,0[$ & ]0, 0.3[ & {$[0.3,0.7[$} & {$[0.7,1]$} \\
\hline $0.0 \%$ & $0.0 \%$ & $52.4 \%$ & $47.6 \%$ & $0.0 \%$ & $0.0 \%$ \\
\hline$N m \mathrm{~F} 2, H \mathrm{~F} 2$ & & Min & -0.62 & $\operatorname{Max}$ & 0.23 \\
\hline$[-1,-0.7]$ & ]$-0.7,-0.3]$ & ]$-0.3,0[$ & ] $0,0.3[$ & {$[0.3,0.7[$} & {$[0.7,1]$} \\
\hline $0.0 \%$ & $\underline{5.2} \%$ & $47.4 \%$ & $47.5 \%$ & $0.0 \%$ & $0.0 \%$ \\
\hline$h m \mathrm{~F} 2, H \mathrm{~F} 2$ & & Min & -0.20 & Max & 0.63 \\
\hline$[-1,-0.7]$ & ]$-0.7,-0.3]$ & ]$-0.3,0[$ & ]0, 0.3[ & {$[0.3,0.7[$} & {$[0.7,1]$} \\
\hline $0.0 \%$ & $0.0 \%$ & $47.3 \%$ & $48.1 \%$ & $4.6 \%$ & $0.0 \%$ \\
\hline
\end{tabular}

indirectly obtained from IRI07 without input of F3/C measurements. However, the obtained results are in agreement with other correlation studies for the F2 key parameters that took ionosonde data into account: for instance, with Liu et al. (2006) and Zhang et al. (2006), who stated, based on ionogram studies at low-latitude regions, that the scale height around the F2 layer peak shows a moderate positive correlation with $h m \mathrm{~F} 2$. It is important to note that $H \mathrm{~F} 2$ is determined only from bottomside measurements below the peak in the case of using ionosonde data.

Furthermore, Lee and Reinisch (2007) studied HF2 variations from ionograms measured at the Jicamarca ionosonde station at $\lambda=283.2^{\circ}$ and $\varphi=-12^{\circ}$ close to the dip equator and also located inside our study area during quiet solar conditions, and found high correlations between $h m \mathrm{~F} 2$ and $H \mathrm{~F} 2$. It should be kept in mind that these correlations are latitude dependent and following Lee and Reinisch (2007), they are more obvious close to the dip equator than in low latitudes. However, correlations between $h m \mathrm{~F} 2$ and $H \mathrm{~F} 2$ in general indicate the influence of the same physical processes on $h m \mathrm{~F} 2$ and $H \mathrm{~F} 2$ variations (Liu et al., 2006).

Negative correlations between $N m \mathrm{~F} 2$ and $H \mathrm{~F} 2$ appear in Fig. 2, where the minimum yields -0.62 . Of the corresponding correlations, $5.2 \%$ can be found in the ] $-0.7,-0.3$ ] interval. Zhang et al. (2006) and Lee and Reinisch (2007) reported the existence of only marginal correlations between the critical frequency $f_{0} \mathrm{~F} 2$ and $H \mathrm{~F} 2$ in low latitudes. Based 
on the relation $N m \mathrm{~F} 2\left[\mathrm{~m}^{-3}\right]=1.24 \times 10^{10} \cdot\left(f_{0} \mathrm{~F} 2\right)^{2}[\mathrm{MHz}]$ (Bilitza, 2002), the maximum electron density is linearly dependent on $(f o \mathrm{~F} 2)^{2}$. Poor correlations should consequently also exist between $N m \mathrm{~F} 2$ and $H \mathrm{~F} 2$.

In our model, approximate values for $H \mathrm{~F} 2$ are directly retrieved from $H \mathrm{~F} 2=\tau / 4.13$ including the slab thickness $\tau$. Correlations between the plasma- or topside scale height and $\tau$ have been found in Stankov and Jakowski (2005) by analyzing CHAMP ionospheric radio occultation data. In addition, studies of Zhang et al. (2006) with ionosonde data showed very high correlations between $\tau$ and HF2, supporting the validity of the retrieval method. $\tau$ is obtained from $\tau=\mathrm{VTEC} / N m \mathrm{~F} 2$. Following Kouris et al. (2008), the correlation between VTEC and $N m \mathrm{~F} 2$ is also certainly very high, i.e., more than 0.9 but not 1 . This eventually causes the remaining negative correlations between $N m \mathrm{~F} 2$ and $H \mathrm{~F} 2$. Liu et al. (2006) found weak negative correlations between $f_{0} \mathrm{~F} 2$ and $H \mathrm{~F} 2$ with local time dependency from studies with ionosonde data ionosonde data of a station located in Wuhan, i.e., in low latitude at $\lambda=114.4^{\circ}, \varphi=30.6^{\circ}$.

In this simulation, the preliminary model of Eq. (3) with the ability to handle scenarios without gaps has been considered. However, reality looks different, showing a sparse and inhomogeneous distribution of measurements. Therefore, the investigation is continued in the following by applying F3/C EDPs.

\section{Real data investigation}

According to the simulated scenario described in Sect. 4, the South American region covering a study area between $\left[250^{\circ}\right.$, $\left.340^{\circ}\right]$ longitude and $\left[-60^{\circ}, 30^{\circ}\right]$ latitude has also been selected for investigations with real F3/C data. Here, two time frames of 24 h, 1 July 2008 (Sect. 7) during quiet solar conditions and 1 July 2012 (Sect. 8) at increased solar activity, have been selected for studying correlations of B-spline coefficients and F2 Chapman parameters. The mean Kp index on 1 July 2008 is 1.1 with a sunspot number of 0 , which indicates quiet conditions as a good starting point for these studies. For 1 July 2012 an increased solar activity with a mean $\mathrm{Kp}$ index of 3.3 and a sunspot number of 84 has been determined. The computation of the mean $\mathrm{Kp}$ here is based on indices and fractional parts provided in $3 \mathrm{~h}$ intervals by the National Geophysical Data Center of National Oceanic and Atmospheric Administration (NOAA, http://www.noaa. gov). The sunspot number refers to the Brussels International Sunspot Number.

The pre-processed F3/C electron density profiles kindly provided by the Center of Space and Remote Sensing Research (CSRSR, http://www.csrsr.ncu.edu.tw) of the National Taiwan University (NTU) (Tsai et al., 2001; Tsai and Tsai, 2004; Tsai et al., 2009) are considered as input data. The removal of outliers and corrupted profiles by means of a rather conservative data screening process has been accom- plished within an additional pre-processing step. The process mainly comprises the following:

- the detection of larger jumps, here $>50 \mathrm{~km}$, in the altitudes between consecutive $N_{\mathrm{e}}$ measurements of a specific EDP;

- the verification of data availability around the peak region within $\pm 50 \mathrm{~km}$ to guarantee a proper description of the profile shape in the peak region;

- the screening of $h m \mathrm{~F} 2$ to be located within the physically reasonable altitude interval of [150 km, $500 \mathrm{~km}]$.

Furthermore, only profiles that reach a minimum altitude below $250 \mathrm{~km}$ and maximum altitude above $500 \mathrm{~km}$ are taken into account to allow for a rather complete description of the vertical $N_{\mathrm{e}}$ distribution in the F2 region. One drawback of the simple mathematical model given by Eq. (1) is the neglectionof an E layer which led in some cases to mismodeling effects (see Limberger et al., 2013). The E layer is typically located in an altitude interval between 90 and $130 \mathrm{~km}$, and therefore only measurements above $150 \mathrm{~km}$ are taken into account. The consideration of the F1 layer has been neglected in this model. The F1 manifestation may arise during daytime at approximately $150-220 \mathrm{~km}$ above Earth and merges with the F2 layer at night. However, the intensity of ionization in the F1 region is generally less than in the E region, and significant features in the used EDPs due to F1 occurrence have not been monitored.

Based on the retrieval concept to derive EDPs from ionospheric radio occultation measurements, the position of each $N_{\mathrm{e}}$ value coincides with the closest point to Earth located on the line of sight between GPS and F3/C at the corresponding observation epoch. Therefore, the time and position of the measurements can vary during an occultation event by several minutes and up to about $10^{\circ}$ in some cases. The derived profile describes the $N_{\mathrm{e}}$ distribution along a slant direction. The determination of F2 Chapman parameters in our model benefits primarily from observations in the peak region. In order to reference the observed profile to a fixed location and thus stabilize the parameter estimation, we assume the verticality of the profiles at latitude, longitude and time of the F2 peak measurement, i.e., the observed $N m \mathrm{~F} 2$. Another characteristic of the retrieval concerns the availability of much denser observations at higher altitudes. These observations, however, contain only limited information about the F2 peak quantities. In order to reduce their influence, measurements above $650 \mathrm{~km}$ are neglected here.

Finally, a total of $123 \mathrm{~F} 3 / \mathrm{C}$ profiles (52\% rejected) for 1 July 2008 and $96 \mathrm{~F} 3 / \mathrm{C}$ profiles (38\% rejected) for 1 July 2012 remain above the target region in South America after the data screening. Most of the rejected profiles have been removed because of the high measurement noise, due to incompleteness or because they are affected by a dominant E layer. It thus becomes clear that a decreasing number of rejections can be expected if an E layer model is introduced. 


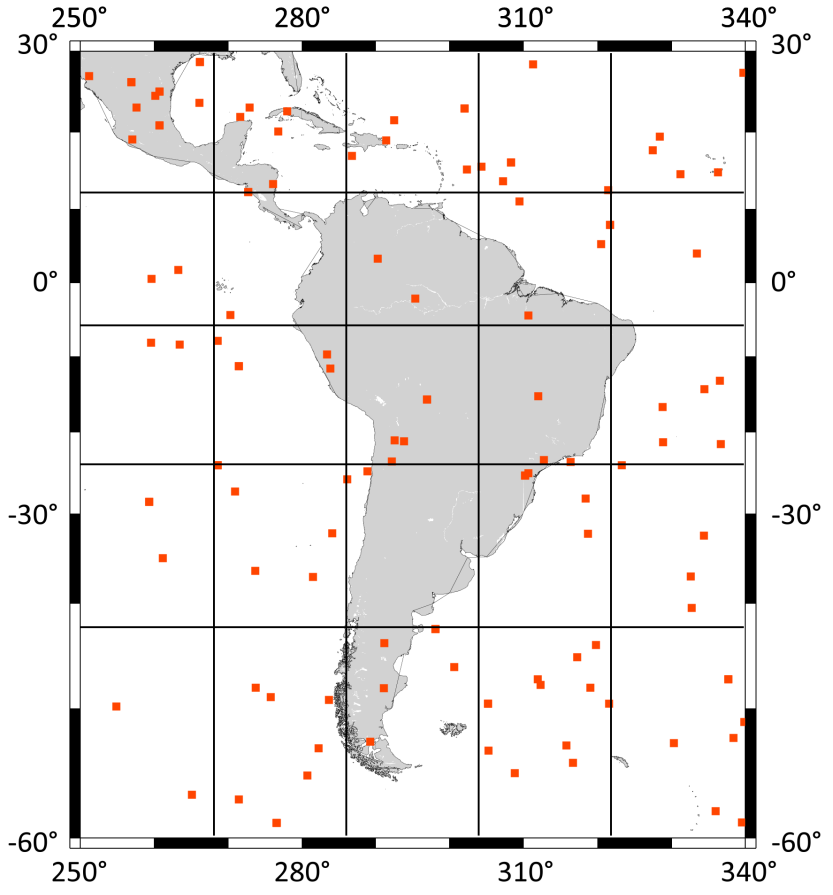

Figure 3. Spatial F3/C EDP distribution observed on 1 July 2008 over the study area in South America.

The distribution of all profiles for 1 July 2008, here independent of the corresponding measurement epoch, is depicted in Fig. 3, and gives a first impression of the sparsity and inhomogeneity of the data. The distribution of profiles for 2012 is comparable and not shown here.

It should be noted that the payload of each F3/C satellite includes two precise orbit determination (POD) and two occultation (OCC) antennas, all of which can be used for the retrieval of EDPs from radio occultations, i.e., different profiles from several antenna may be acquired for the same location and time. Hence, the number of profiles is larger than the number of orange squares in Fig. 3. Furthermore, although the number of input profiles in 2008 is slightly higher than 2012 , the sparsity of the distribution is not significantly improved.

To derive a combined graphical relation between the localization of the EDPs with their corresponding measurement epoch, Fig. 3 has been firstly subdivided into $18^{\circ} \times 18^{\circ}$ wide cells. Afterwards, the $24 \mathrm{~h}$ investigation time frame has been divided into $2 \mathrm{~h}$ intervals and all profiles belonging to a specific spatiotemporal cell were counted. This situation is depicted in Fig. 4 as a combination of a graphical and numerical expression and gives a good impression of the data distribution.

Again, as also depicted in Fig. 3, the spatial $18^{\circ} \times 18^{\circ}$ cells are visible. Each cell contains a clock symbol, indicated by the centrally positioned black circle with a green background, that represents $12 \mathrm{~h}$ divided into $2 \mathrm{~h}$ segments. Two numerical values refer to each $2 \mathrm{~h}$ segment, the first one

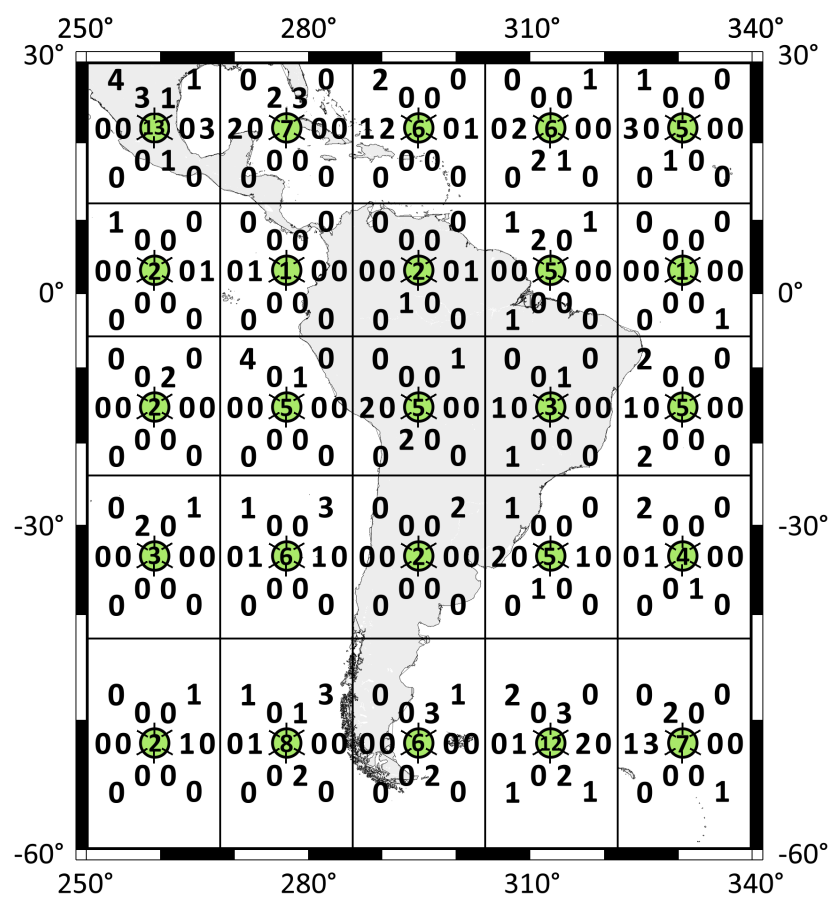

Figure 4. F3/C EDP distribution related to spatiotemporal grid cells observed on 1 July 2008 in the South America region.

on the inner ring and the second one on the outer ring. The inner value refers to the time between 00:00 and 12:00 UT, and numbers on the outer ring are related to a $2 \mathrm{~h}$ segment between 12:00 and 24:00 UT. Additionally, the number inside the clock denotes the total number of profiles observed in this specific cell, which equals the number of profiles in the corresponding cell of Fig. 3 (counting the numbers would consequently result in the total of 123 EDPs observed during this day). The first cell of the second row, for instance, covers the region of -6 to $12^{\circ}$ latitude and 250 to $268^{\circ}$ longitude. Altogether it contains two profiles: one which has been observed between 14:00 and 16:00 UT (outer circle, east side) and the second one between 22:00 and 24:00 UT (outer circle, northwest).

The total number of profiles measured within each cell spans from 1 to 13 and shows that the B-spline levels with respect to longitude and latitude have to be chosen rather low in order to bridge those regions without data. This situation is also visible from Fig. 3, where some cells are nearly empty or the profiles are at least located close to the cell border. Therefore we decided to adapt an average spatial distance between profiles of around $18^{\circ}$ latitude and longitude according to the cell width. Depending on this assessment, the relation

$J \leq \log _{2}\left(\frac{\mathrm{si}_{\max }-\mathrm{si}_{\min }}{\Delta \mathrm{si}}-1\right)$

with the sampling $\Delta \mathrm{si}$ and boundaries $\mathrm{si}_{\min }$ and $\mathrm{si}_{\max }$ has been applied to estimate a suitable B-spline level. Equation (4) was proposed by Schmidt et al. (2011) and leads to 


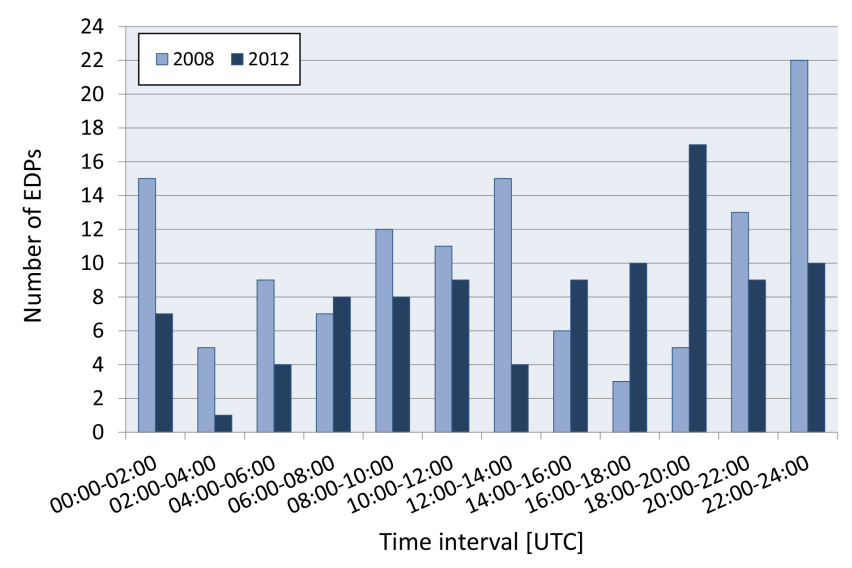

Figure 5. Temporal data distribution: EDPs are counted within $2 \mathrm{~h}$ intervals for 1 July 2008 (light blue) and 1 July 2012 (dark blue).

$J_{\varphi} \leq 2, J_{\lambda} \leq 2 \longrightarrow J_{\varphi}=J_{\lambda}=2$. Hence, the B-spline level has been adapted to the situation by selecting $J_{\lambda}=J_{\varphi}=$ 2 , meaning that the number of B-splines yields $K\left(J_{\varphi}\right)=$ $K\left(J_{\lambda}\right)=6$ in latitude and longitude directions.

To find an adequate level for the time, all profiles belonging to a specific time segment in Fig. 4, independent of the position, have been counted and plotted in Fig. 5, which demonstrates the sparsity of the data distribution in time.

For instance, there are only three profiles available between 16:00 and 18:00 UT in 2008 and just one between 02:00 and 04:00 UT in 2012. Based on this distribution, an approximate time sampling of $2.5 \mathrm{~h}$ has been chosen, and, according to Eq. (4), this leads to a B-spline level of $J_{t} \leq 3.1 \longrightarrow J_{t}=3$, resulting in $K\left(J_{t}\right)=10$ splines with respect to time. The B-spline levels found here have also been considered for the studies of 2012.

\section{Extended adjustment system}

In order to process sparse and inhomogeneous data, the adjustment system introduced with Eq. (3) has to be extended to allow for the bridging of data gaps in a reliable way with prior information. The extended normal equations yield

$\widehat{\boldsymbol{d}}=\boldsymbol{d}_{0}+\left(\frac{\boldsymbol{A}^{T} \mathbf{P} \boldsymbol{A}}{\widehat{\sigma}_{\mathrm{F} 3 / \mathrm{C}}^{2}}+\mathbf{W}\right)^{-1}\left(\frac{\boldsymbol{A}^{T} \mathbf{P} \boldsymbol{L}}{\widehat{\sigma}_{\mathrm{F} 3 / \mathrm{C}}^{2}}+\mathbf{W} \boldsymbol{M}\right)$,

where $\boldsymbol{M}_{\mathrm{it}}=\boldsymbol{\mu}-\widehat{\boldsymbol{d}}_{\mathrm{it}-1}$ is computed from the difference between the prior information vector $\boldsymbol{\mu}$ and the coefficient solution of the previous iteration it -1 in order to constrain the solution in regions without observations to the prior information. Here, the prior information vector $\boldsymbol{\mu}$ (just like the initial coefficients $\boldsymbol{d}_{0}$ ) is derived in a pre-processing step from IRI07, which is considered as the background model, but it should be noted that the data sources for initial and prior information need not necessarily be the same. $\mathbf{W}$ is an extended weight matrix of the prior information defined by

$\mathbf{W}=\operatorname{diag}\left(\frac{\mathbf{P}_{N m \mathrm{~F} 2}}{\widehat{\sigma}_{N m \mathrm{~F} 2}^{2}}, \frac{\mathbf{P}_{h m \mathrm{~F} 2}}{\widehat{\sigma}_{h m \mathrm{~F} 2}^{2}}, \frac{\mathbf{P}_{H \mathrm{~F} 2}}{\widehat{\sigma}_{H \mathrm{~F} 2}^{2}}\right)$

with a block diagonal structure involving the individual weight matrices $\mathbf{P}_{N m \mathrm{~F} 2}, \mathbf{P}_{h m \mathrm{~F} 2}$ and $\mathbf{P}_{H \mathrm{~F} 2}$ of the key parameter-related coefficients. Advanced information on dependencies between observations or key parameters, as well as knowledge allowing for an individual weighting, was not available, and therefore identity matrices were introduced.

Equation (5) considers variance factors for $\mathrm{F} 3 / \mathrm{C}\left(\sigma_{\mathrm{F} 3 / \mathrm{C}}^{2}\right)$ and the prior information groups $\left(\sigma_{N m \mathrm{~F} 2}^{2}, \sigma_{h m \mathrm{~F} 2}^{2}, \sigma_{H \mathrm{~F} 2}^{2}\right)$ by means of $\mathbf{W}$. These variance components serve as relative weighting factors and are determined within a variance component estimation (VCE) (Koch, 1999; Koch and Kusche, 2002). The system does not account for covariance factors, meaning that no correlations between parameter groups or observations are taken into account. From the availability of a dense, homogenous measurement distribution - basically without data gaps - it can be expected that the VCE strongly decreases the influence of the prior information since no background data are required to solve for the unknown coefficient corrections. This in fact occurred for the simulation in Sect. 4, where increased correlations occurred while no prior information was involved. For a more realistic scenario the situation is typically dominated by an inhomogeneous, sparse measurement distribution and the system iteratively adapts the prior information weight in order to balance with the observation weight to bridge the data gaps. This should be kept in mind since possible information stemming from the observations may be suppressed in a scenario with a relatively high prior information weight. Especially concerning the covariance factors which have not been considered in the stochastic model so far, the relevant correlation information cannot be retrieved.

The resulting correlation matrices will still be denoted $\mathbf{K}_{x x}$ and $\mathbf{K}_{t t}$. For further details on the estimation procedure for coefficients and variance components we refer the reader to Limberger et al. (2013). The modeling concept presented here is based on the idea of improving existing background information in regions where observations are available and bridging data gaps with prior information wherever necessary. The extent of the corrected regions depends on the chosen B-spline levels, which are typically adapted to the average measurement density.

\section{July 2008: low solar activity}

The studies on 1 July 2008 include a correlation analysis on the B-spline series coefficient level $\left(\mathbf{K}_{x x}\right)$, followed by investigations on the key parameter correlations $\left(\mathbf{K}_{t t}\right)$ and finally a section about the matrix composition structure. 


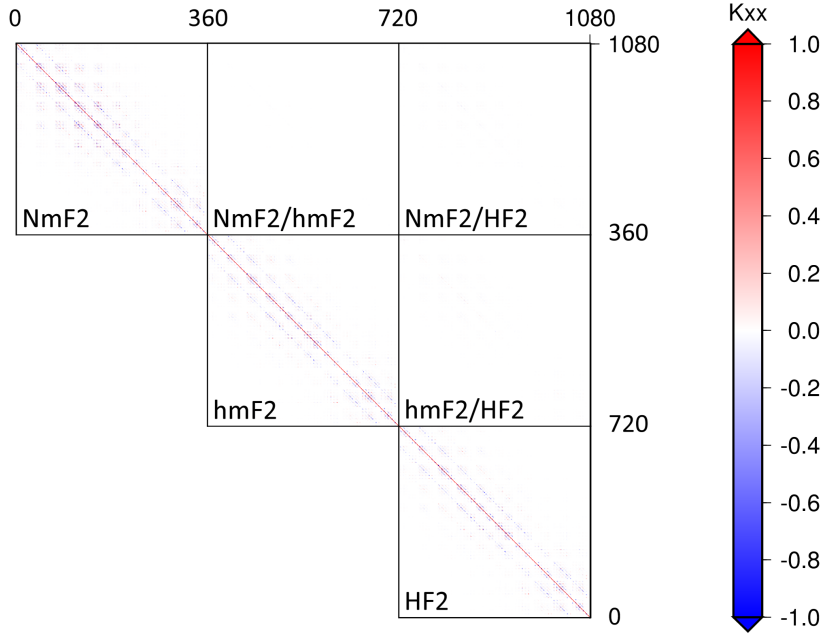

Figure 6. Correlation matrix of the coefficients $\mathbf{K}_{x x}$ for 1 July 2008. Due to the symmetry of the matrices, only those block submatrices belonging to the upper triangular structure have been depicted.

\subsection{Coefficient correlations, $\mathbf{K}_{x x}$}

The correlation matrix of the coefficients $\mathbf{K}_{x x}$, computed for 1 July 2008, is given in Fig. 6.

It has previously been stated in Sect. 6 that key parameter covariances cannot be extracted from this system since they are not considered in the stochastical model and observationbased correlations may be suppressed because of strong prior information. This effect becomes obvious from the correlation matrix $\mathbf{K}_{x x}$ in Fig. 6 with the absence of correlations between coefficients related to the different key parameters. The distribution of $\mathrm{F} 3 / \mathrm{C}$ measurements is inhomogeneous and sparse, which consequently leads to an increased a priori information weighting and depression of interparameter correlations. It can be vaguely discerned that only correlations originating from adjacent B-splines appear close to the diagonal of $\mathbf{K}_{x x}$ as a mixture of characteristic positive and negative dependencies due to the B-spline nature. According to the approach in Sect. 4, the correlation structure should be amplified by the step from $\mathbf{K}_{x x}$ to $\mathbf{K}_{t t}$.

\subsection{Key parameter correlations, $\mathbf{K}_{t t}$}

As can be seen from Fig. 7, noticeable blue/red-colored patches along the diagonal become visible, identifying correlations between nearby key parameters. The width of these patches depends on the resolution of the computed $\mathbf{K}_{t t}$ matrix. Here, a spatial resolution of $5^{\circ} \times 5^{\circ}$ has been chosen.

The corresponding numerical information for $\mathbf{K}_{t t}$ is provided in Table .

Total minimum and maximum correlations are close to \pm 1 , reflecting, in particular, the linear dependencies between neighboring key parameters which are highly correlated due to the low B-spline level. This can also be interpreted from

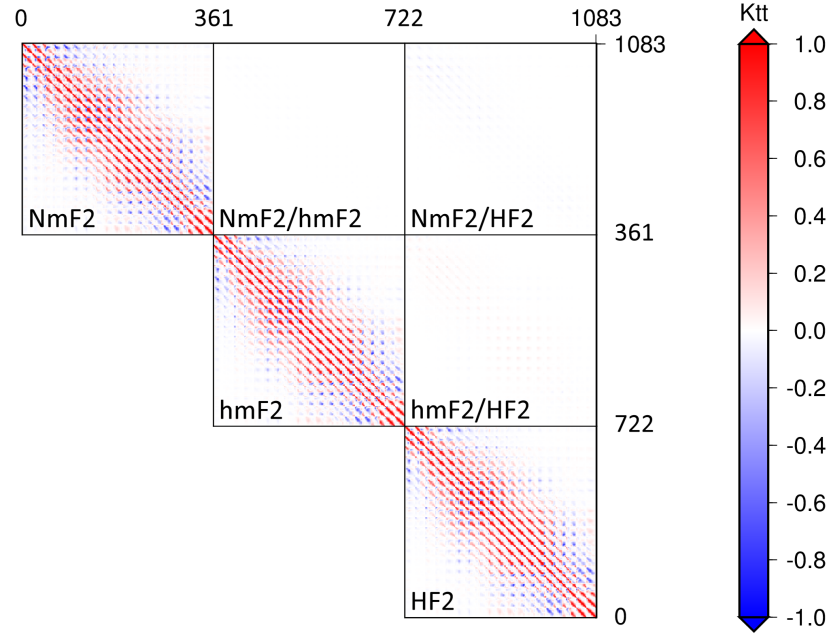

Figure 7. Correlation matrices of the key parameters $\mathbf{K}_{t t}$ for the fixed time moment on 1 July 2008, 12:00 UT. $\mathbf{K}_{t t}$ represents correlations for parameters related to a $\Delta \varphi=5^{\circ} \times \Delta \lambda=5^{\circ}$ grid.

Table 2. Key parameter correlations contained in $\mathbf{K}_{t t}$ for 1 July 2008. Table is displayed in the same manner as Table 1 .

\begin{tabular}{lrrrrr}
\hline Total $\mathbf{K}_{t t}$ & & Min & -0.89 & Max & 0.99 \\
\hline$N m \mathrm{~F} 2$ & & Min & -0.89 & $\operatorname{Max}$ & 0.99 \\
{$[-1,-0.7]$} & ]$-0.7,-0.3]$ & ]$-0.3,0[$ & {$[0,0.3[$} & {$[0.3,0.7[$} & {$[0.7,1]$} \\
$0.1 \%$ & $1.6 \%$ & $\mathbf{4 3 . 9} \%$ & $\mathbf{4 2 . 9} \%$ & $\underline{7.6} \%$ & $\underline{3.9} \%$ \\
\hline$h m \mathrm{~F} 2$ & & Min & -0.89 & $\operatorname{Max}$ & 0.99 \\
{$[-1,-0.7]$} & ]$-0.7,-0.3]$ & ]$-0.3,0[$ & {$[0,0.3[$} & {$[0.3,0.7[$} & {$[0.7,1]$} \\
$0.0 \%$ & $1.0 \%$ & $\mathbf{4 4 . 7} \%$ & $\mathbf{4 2 . 6} \%$ & $\underline{7.4} \%$ & $\underline{3.9} \%$ \\
\hline$H \mathrm{~F} 2$ & & $\operatorname{Min}$ & -0.89 & $\operatorname{Max}$ & 0.99 \\
{$[-1,-0.7]$} & ]$-0,7,-0.3]$ & ]$-0,3,0[$ & {$[0,0.3[$} & {$[0.3,0.7[$} & {$[0.7,1]$} \\
$0.0 \%$ & $1.0 \%$ & $\mathbf{4 5 . 2} \%$ & $\mathbf{4 2 . 5} \%$ & $\underline{7.3} \%$ & $\underline{3.9} \%$ \\
\hline$N m \mathrm{~F} 2, h m \mathrm{~F} 2$ & & $\operatorname{Min}$ & -0.03 & $\mathrm{Max}$ & 0.01 \\
{$[-1,-0.7]$} & ]$-0.7,-0.3]$ & ]$-0.3,0[$ & {$[0,0.3[$} & {$[0.3,0.7[$} & {$[0.7,1]$} \\
$0.0 \%$ & $0.0 \%$ & $\mathbf{6 0 . 8} \%$ & $\mathbf{3 9 . 2} \%$ & $0.0 \%$ & $0.0 \%$ \\
\hline$N m \mathrm{~F} 2, H \mathrm{~F} 2$ & & $\mathrm{Min}$ & -0.15 & $\mathrm{Max}$ & 0.04 \\
{$[-1,-0.7]$} & ]$-0.7,-0.3]$ & ]$-0.3,0[$ & {$[0,0.3[$} & {$[0.3,0.7[$} & {$[0.7,1]$} \\
$0.0 \%$ & $0.0 \%$ & $\mathbf{6 0 . 8} \%$ & $\mathbf{3 9 . 2} \%$ & $0.0 \%$ & $0.0 \%$ \\
\hline$h m \mathrm{~F} 2, H \mathrm{~F} 2$ & & $\mathrm{Min}$ & -0.05 & $\mathrm{Max}$ & 0.18 \\
{$[-1,-0.7]$} & ]$-0.7,-0.3]$ & ]$-0.3,0[$ & {$[0,0.3[$} & {$[0.3,0.7[$} & {$[0.7,1]$} \\
$0.0 \%$ & $0.0 \%$ & $\mathbf{4 3 . 5} \%$ & $\mathbf{5 6 . 5} \%$ & $0.0 \%$ & $0.0 \%$ \\
\hline
\end{tabular}

those cells with underlined numbers which show significant positive correlations in the $[0.3,0.7[$ and $[0.7,1]$ intervals of $N m \mathrm{~F} 2, h m \mathrm{~F} 2$ and $H \mathrm{~F} 2$.

\subsection{Matrix composition}

Discussions about the correlation matrix structure have been neglected in the context of the simulation (Sect. 4) and will be explained in detail here. The upper triangle structure of the $h m \mathrm{~F} 2$ submatrix from Fig. 7 is depicted in Fig. 8. The submatrices for $N m \mathrm{~F} 2$ and $H \mathrm{~F} 2$ are structured in a similar way. 


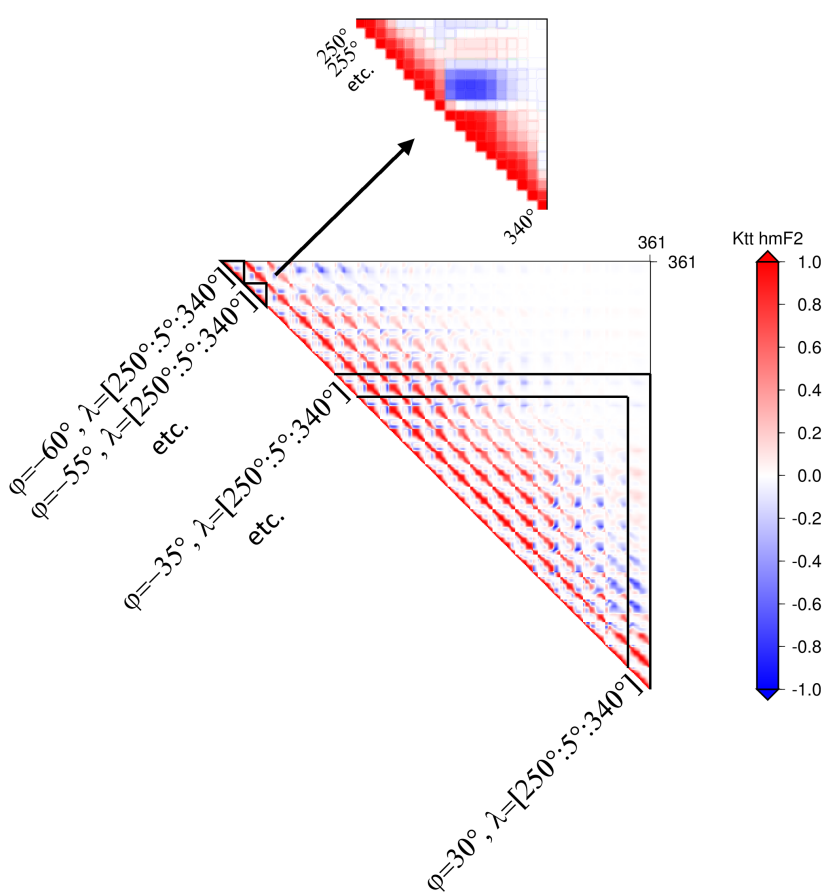

Figure 8. Upper triangular structure of the $h m \mathrm{~F} 2$ block submatrix located on the diagonal of Fig. 7 showing the internal $h m \mathrm{~F} 2$ key parameter correlations for the fixed time moment of 1 July 2008, 12:00 UT. The entries are related to a $\Delta \varphi=5^{\circ} \times \Delta \lambda=5^{\circ}$ grid.

The numbering of the matrix elements in $\mathbf{K}_{t t}$ follows the order of longitude in the innermost loop and latitude in the outermost. Figure 7 is given as a snapshot at 12:00 UT, and therefore temporal correlations are not visible. For clarification, tags have been added in Fig. 8 to describe the arrangement of the diagonal elements, with each tag referring to a specific latitude position. Two small triangular-shaped areas have been framed to group all elements belonging to a specific latitude position. Consequently, pixels within such a triangle describe the longitude correlations of $h m \mathrm{~F} 2$. As an example, the correlations between $\lambda=250^{\circ}$ and $\lambda=340^{\circ}$ at $\varphi=-55^{\circ}$ have been zoomed in on to give a detailed representation. Here, every pixel along the diagonal refers to a longitude position. It can be seen that, due to the $5^{\circ}$ separation between key parameters, the diagonal consists of 19 elements. It becomes clear that the longitude correlations are very large for adjacent elements and that interior elements show a large negative correlation, indicated by the blue region. The same pattern appears multiple times in Fig. 8 and can be better explained by considering the spline distribution depicted in Fig. 9 as an example for latitude.

With regard to the investigation area covering $90^{\circ} \times 90^{\circ}$, it can be inferred that the distance between neighboring Bspline peaks is around $20^{\circ}(\approx 2000 \mathrm{~km})$ in latitude and longitude direction for the defined levels $J_{\lambda}=J_{\varphi}=2$. This applies at least for the "regular" splines in the interior region and is different for the two first and two last endpoint-

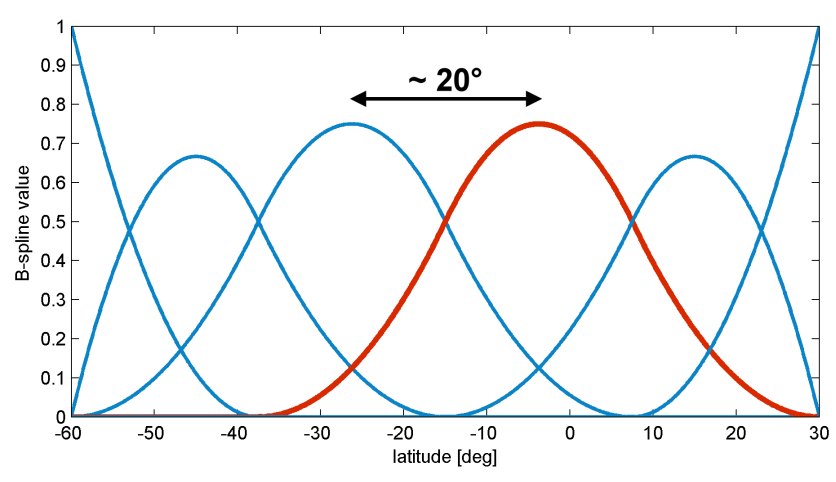

Figure 9. 1-D representation of endpoint-interpolating polynomial B-splines of level $J=2\left(K_{J}=6\right)$ with respect to a latitude interval between $-60^{\circ}$ and $30^{\circ}$. The distance between neighboring, "regular" spline peaks is approximately $20^{\circ}$.

interpolating splines. (For completeness, $K\left(J_{t}\right)=10$ splines are distributed over time in the interval of $24 \mathrm{~h}$ and spline peaks are placed around every $160 \mathrm{~min}$.) Figure 9 represents the spline distribution in the latitude direction with a peak separation of about $20^{\circ}$.

A single spline function, e.g., the red-colored spline $\phi_{2}^{4}$ in Fig. 9, is again illustrated in Fig. 10a and shows that the nonzero interval for a regular interior spline based on level $J_{\varphi}=2$ covers approximately $65^{\circ}$ in a total interval of $90^{\circ}$. Again, it should be kept in mind that the boundary splines are exceptions. For this scenario, $J_{\lambda}$ and $J_{\varphi}$ are chosen equal and consequently the influence zone spanned by the latitude and longitude axis becomes circular (diameter yields around $65^{\circ}$ ), indicated by the bird's eye view perspective in Fig. $10 \mathrm{~b}$.

Under consideration that the two interior splines together already cover the whole region, a negative correlation may appear between them and consequently also between key parameters located in the interior region. This relation can clearly be seen from the longitude correlations in Fig. 8. The effect, however, is not as obvious for the latitude correlations.

Additionally, two black bands related to $\varphi=-35^{\circ}$ and $\varphi=30^{\circ}$ are depicted in relation to the knowledge that a single spline already covers more than two-thirds of the region and, following Fig. 10a (or also Fig. 10b), approximately $65^{\circ}$. The band therefore shows how the correlations evolve between directly neighboring and up to $65^{\circ}$ separated parameters. A steadily decreasing trend is visible until the correlation converges to zero towards the intersection of both bands where the distance between the parameters reaches $65^{\circ}$ in the latitude direction.

The appearance of internal correlations between the variables of a specific key parameter resulting from the B-spline modeling approach is expected and not surprising. The absence of interparameter dependencies in this scenario can be explained by studying the observation and parameter weighting resulting from the VCE. The normal equations are given by Eq. (5), where variance components for $\mathrm{F} 3 / \mathrm{C}$ and the prior 

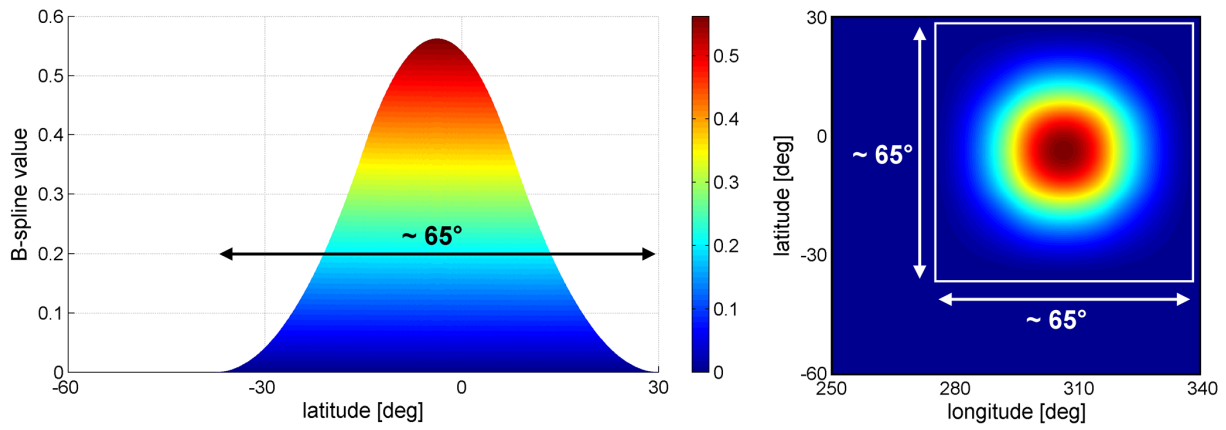

Figure 10. Representation of a single B-spline with nonzero interval of $\approx 65^{\circ}$ (a) and corresponding bird's eye view perspective giving the extent of the spline function in latitude and longitude (b).

information, i.e., a global variance factor for each group of a priori coefficients with respect to $N m \mathrm{~F} 2, h m \mathrm{~F} 2$ and $H \mathrm{~F} 2$, are included. All observations and the prior information are equally weighted, i.e., $\mathbf{P}$ and $\mathbf{P}_{N m \mathrm{~F} 2}, \mathbf{P}_{h m \mathrm{~F} 2}$ as well as $\mathbf{P}_{H \mathrm{~F} 2}$ are introduced as identity matrices, while the VCE controls the relative weighting between $\mathrm{F} 3 / \mathrm{C}$ and the prior information. Following the investigations in Sect. 5, the EDP distribution is very sparse and the adjustment system has to deal with large data gaps. Hence, the ratio between COSMIC and the prior information weighting is rather small due to a small $\sigma_{h m \mathrm{~F} 2}$ (or also $\sigma_{N m \mathrm{~F} 2}$ and $\sigma_{H \mathrm{~F} 2}$ ) in comparison to $\sigma_{\mathrm{F} 3 / \mathrm{C}}-$ and consequently a relatively high prior information weighting is considered. In other words, the diagonal elements of the extended weight matrix $\mathbf{W}$, given by Eq. (6), contribute large values which are added to the diagonal of the normal equations matrix $\mathbf{N}$ in Eq. (5). The influence of $\mathbf{W}$ on the normal equations is therefore very strong and suppresses the occurrence of correlations originating from the observations. In addition, the stochastic model introduced here is incomplete and currently does not account for covariances between key parameters due to the fact that only diagonal weighting matrices have been introduced. Stochastic correlations therefore cannot appear. The absence of parameter correlations is confirmed by Figs. 6 and 7 .

\section{1 July 2012: Increased solar activity}

To prove the general validity of the conclusions obtained for the 1 July 2008 scenario during quiet solar conditions, the same procedure has been conducted for 1 July 2012, a day with slightly higher solar activity.

All settings for the studies of 1 July 2012 are maintained as for 2008 - the configuration for the data screening, B-spline levels and computation sequence - as explained in Sects. 5 and 7. A detailed description of the matrix composition for the coefficient and key parameter correlation matrices $\mathbf{K}_{x x}$ and $\mathbf{K}_{t t}$, respectively, has already been given in Sect. 7.

The results are comparable to the outcomes of 2008 and have not manifested any differences. Studies on the coef-

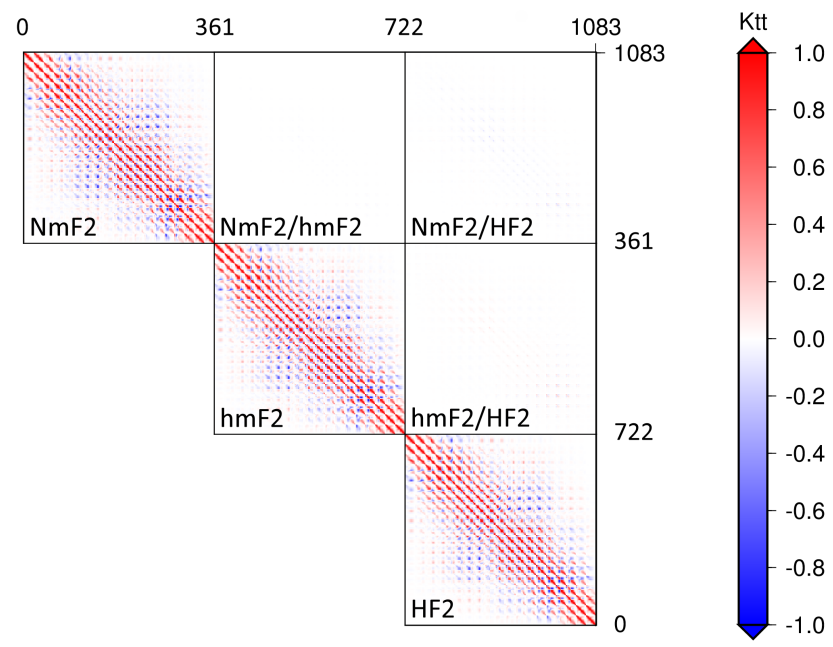

Figure 11. Correlation matrix of the key parameters $\mathbf{K}_{t t}$ for the fixed time moment on 1 July 2012, 12:00 UT. $\mathbf{K}_{t t}$ represents correlations for parameters related to a $\Delta \varphi=5^{\circ} \times \Delta \lambda=5^{\circ}$ grid

ficient level are therefore neglected here and only $\mathbf{K}_{t t}$ as well as the corresponding numerical values of the correlation distribution will be studied at this point. $\mathbf{K}_{t t}$ is depicted in Fig. 11 and clearly shows a similar pattern to Fig. 7.

In accordance with Table, a numerical expression of the correlations is provided in Table . Again, the majority of correlations are located within the ] $-0.3,0$ [ and [0,0.3[ intervals. Around 9.3 and $4.7 \%$ of the correlations for the single parameters have been detected in the $[0.3,0.7[$ and $[0.7,0.1]$ intervals, respectively. The results here again confirm the absence of correlations between key parameters resulting from the combination of the applied model approach with a sparse and inhomogeneous measurement distribution.

\section{Conclusions}

The investigations performed in this paper are provided as an extension of Limberger et al. (2013) and were strongly fo- 
Table 3. Key parameter correlations contained in $\mathbf{K}_{t t}$ for 1 July 2012. Table is displayed in the same manner as Table 1 .

\begin{tabular}{lrrrrr}
\hline Total $\boldsymbol{K}_{t t}$ & & Min & -0.27 & Max & 0.99 \\
\hline$N m \mathrm{~F} 2$ & & Min & -0.26 & Max & 0.99 \\
{$[-1,0.7]$} & ]$-0.7,-0.3]$ & ]$-0.3,0[$ & {$[0,0.3[$} & {$[0.3,0.7[$} & {$[0.7,1]$} \\
$0.0 \%$ & $0.0 \%$ & $\mathbf{3 5 . 0} \%$ & $\mathbf{5 1 . 1} \%$ & $\underline{9.3} \%$ & $\underline{4.7} \%$ \\
\hline$h m \mathrm{~F} 2$ & & Min & -0.27 & $\operatorname{Max}$ & 0.99 \\
{$[-1,0.7]$} & ]$-0.7,-0.3]$ & ]$-0.3,0[$ & {$[0,0.3[$} & {$[0.3,0.7[$} & {$[0.7,1]$} \\
$0.0 \%$ & $0.0 \%$ & $\mathbf{3 5 . 6} \%$ & $\mathbf{5 0 . 5} \%$ & $\underline{9.3} \%$ & $\underline{4.7} \%$ \\
\hline$H \mathrm{~F} 2$ & & $\mathrm{Min}$ & -0.26 & $\mathrm{Max}$ & 0.98 \\
{$[-1,0.7]$} & ]$-0,7,-0.3]$ & ]$-0,3,0[$ & {$[0,0.3[$} & {$[0.3,0.7[$} & {$[0.7,1]$} \\
$0.0 \%$ & $0.0 \%$ & $\mathbf{3 5 . 8} \%$ & $\mathbf{5 0 . 3} \%$ & $\underline{9.3} \%$ & $\underline{4.7} \%$ \\
\hline$N m \mathrm{~F} 2, h m \mathrm{~F} 2$ & & $\mathrm{Min}$ & -0.00 & $\mathrm{Max}$ & 0.00 \\
{$[-1,0.7]$} & ]$-0.7,-0.3]$ & ]$-0.3,0[$ & {$[0,0.3[$} & {$[0.3,0.7[$} & {$[0.7,1]$} \\
$0.0 \%$ & $0.0 \%$ & $\mathbf{7 7 . 2} \%$ & $\mathbf{2 2 . 8} \%$ & $0.0 \%$ & $0.0 \%$ \\
\hline$N m \mathrm{~F} 2, H \mathrm{~F} 2$ & & $\mathrm{Min}$ & -0.02 & $\mathrm{Max}$ & 0.00 \\
{$[-1,0.7]$} & ]$-0.7,-0.3]$ & ]$-0.3,0[$ & {$[0,0.3[$} & {$[0.3,0.7[$} & {$[0.7,1]$} \\
$0.0 \%$ & $0.0 \%$ & $\mathbf{7 4 . 6} \%$ & $\mathbf{2 5 . 4} \%$ & $0.0 \%$ & $0.0 \%$ \\
\hline$h m \mathrm{~F} 2, H \mathrm{~F} 2$ & & $M i n$ & -0.00 & $\operatorname{Max}$ & 0.02 \\
{$[-1,0.7]$} & ]$-0.7,-0.3]$ & ]$-0.3,0[$ & {$[0,0.3[$} & {$[0.3,0.7[$} & {$[0.7,1]$} \\
$0.0 \%$ & $0.0 \%$ & $\mathbf{3 1 . 9} \%$ & $\mathbf{6 8 . 1} \%$ & $0.0 \%$ & $0.0 \%$ \\
\hline
\end{tabular}

cused on studies of correlations between F2 Chapman key parameters $N m \mathrm{~F} 2, h m \mathrm{~F} 2$ and $H \mathrm{~F} 2$ based on the usage of endpoint-interpolating polynomial B-splines in a regional modeling approach. A simulated and a real data scenario were taken into account. One day during quiet solar conditions on 1 July 2008 and another one in the solar maximum period on 1 July 2012 have been considered. In addition to studies on the dependencies between the B-spline series coefficients referring to a specific key parameter, interdependencies between F2 Chapman key parameters have also been analyzed. After comparisons between the simulated scenario (dense, homogenous data grid) and the real data scenario (sparsely, inhomogeneously distributed F3/C EDPs), it can be concluded that the chosen preliminary stochastic model supports the separability of the different types of series coefficients regarding the use of sparse and inhomogeneously distributed input data. The stochastic model effectively causes a decorrelation by suppressing key parameter correlations and enables the reliable estimation of the key parameters by increasing the prior information weight by means of the VCE as a consequence of the sparse $\mathrm{F} 3 / \mathrm{C}$ measurement distribution. However, there are enough F3/C input data to correct the background information, as shown by Limberger et al. (2013) and Liang et al. (2014). Another important point in this context concerns the incomplete stochastic model where no covariances between key parameters have been considered so far. In combination with other observation techniques it can be assumed that estimability benefits from the different sensitivities of the techniques, leading to a (desired) downweighting of the prior information. Consequently, the importance of considering key parameter correlations goes along with the number and distribution of further observation tech- niques. Since the simulated scenario with coefficient correlations reaching a minimum of -0.62 between $N m \mathrm{~F} 2$ and $H \mathrm{~F} 2$ (5.2\% of the correlations within the $]-0.7,-0.3$ ] interval) and a maximum of 0.63 between $h m \mathrm{~F} 2$ and $H \mathrm{~F} 2(4.6 \%$ of the correlations within the $[0.3,0.7$ [ interval) was still solvable, the implementation of an extended stochastic model is not urgent but definitely an important task that is in need of discussion in the near future.

Acknowledgements. The authors would like to thank Lung-Chih Tsai from CSRSR at the NCU, Taiwan, for providing the FORMOSAT-3/COSMIC electron density profiles. This work was carried out as part of the project "Multi-scale model of the ionosphere from the combination of modern space-geodetic satellite techniques", which is funded by the German Research Foundation (DFG), Bonn, Germany.

Topical Editor K. Hosokawa thanks J. F. Conte and M. M. Alizadeh for their help in evaluating this paper.

\section{References}

Alizadeh, M. M.: Multi-Dimensional modeling of the ionospheric parameters, using space geodetic techniques, $\mathrm{PhD}$ Thesis, University of Technology, Vienna, Austria, February 2013.

Altadill, D., Magdaleno, S., Torta, J. M., and Blanch, E.: Global empirical models of the density peak height and of the equivalent scale height for quiet conditions, Adv. Space Res., 52: 17561769, 2012.

Angling, M. J.: First assimilations of COSMIC radio occultation data into the Electron Density Assimilative Model (EDAM), Ann. Geophys., 26, 353-359, doi:10.5194/angeo-26-353-2008, 2008.

Azpilicueta, F., Brunini, C., and Radicella, S. M.: Global ionospheric maps from GPS observations using modip latitude, Adv. Space Res., 38, 11, 2324-2331, doi:10.1016/j.asr.2005.07.069, 2006.

Bilitza, D.: International Reference Ionosphere 2000, Radio Sci., 36, 261-275, 2001.

Bilitza, D.: Ionospheric models for radio propagation studies, Review of Radio Science 1999-2002, Oxford University Press, 625-679, 2002.

Bilitza, D., Mckinnell, L. A., Reinisch, B., and Fuller-Rowell, T.: The international reference ionosphere today and in the future, J. Geodyesy, 85, 909-920, 2011.

Brunini, C., Conte, J. F., Azpilicueta, F., and Bilitza, D.: A different method to update monthly median hmF2 values, Adv. Space Res., 51, 2322-2332, 2013.

Davies, K.: Ionospheric radio, Peter Peregrinus Ltd., London, 1990.

Dettmering, D., Schmidt, M., Heinkelmann, R., and Seitz, M.: Combination of different space-geodetic observations for regional ionosphere modeling, J. Geodesy, 85, 989-998, doi:10.1007/s00190-010-0423-1, 2011.

Dettmering, D., Schmidt, M., Limberger, M.: Contributions of DORIS to ionosphere modeling, in: Proceedings of "20 Years of Progress in Radar Altimetry”, edited by: Ouwehand, L., IDS Workshop, Sept. 2012, Venice, Italy, ESA SP-710 (CD-ROM), ISBN 978-92-9221-274-2, ESA/ESTEC, 2013. 
Dow, J. M., Neilan, R. E., and Rizos, C.: The International GNSS Service in a changing landscape of Global Navigation Satellite Systems, J. Geodesy, 83, 191-198, doi:10.1007/s00190-0080300-3, 2009.

Feltens, J.: Chapman profile approach for 3-D global TEC representation, Proceeding of the IGS AC Workshop, 285-297, 1998.

Hernández-Pajares, M., Juan, J. M., Sanz, J., Orus, R., Garcia-Rigo, A., Feltens, J., Komjathy, A., Schaer, S. C., and Krankowski, A.: The IGS VTEC maps: a reliable source of ionospheric information since 1998, J. Geodesy, 83, 263-275, 2009.

Hernández-Pajares, M.: IGS Ionosphere WG Status Report: Performance of IGS Ionosphere TEC Maps, IGS Workshop, Bern, 2004.

Hernández-Pajares, M., Juan, J. M., and Sanz, J.: New approaches in global ionospheric determination using ground GPS data, J. Atmos. Sol.-Terr. Phys., 61, 1237-1247, doi:10.1016/S13646826(99)00054-1, 1999

Hoque, M. M. and Jakowski, N.: A new global model for the ionospheric F2 peak height for radio wave propagation, Ann. Geophys., 30, 797-809, doi:10.5194/angeo-30-797-2012, 2012.

Jakowski, N.: Ionospheric GPS radio occultation measurements on board CHAMP, GPS Solutions, 9, 88-95, doi:10.1007/s10291005-0137-7, 2005.

Jakowski, N., Leitinger, R., and Angling, M.: Radio occultation techniques for probing the ionosphere, Ann. Geophys., 47, doi:10.4401/ag-3285, 2004

Jayachandran, B., Krishnankutty, T. N., and Gulyaeva, T. L.: Climatology of ionospheric slab thickness, Ann. Geophys., 22, 25-33, doi:10.5194/angeo-22-25-2004, 2004.

Kirchengast, G., Foelsche, U., and Steiner, A. K.: Occultations for probing atmosphere and climate, Springer Berlin Heidelberg, ISBN 3-540-22350-9, 2004.

Koch, K. R.: Parameter estimation and hypothesis testing in linear models, Springer, Berlin, 1999.

Koch, K.-R. and Kusche, J.: Regularization of geopotential determination from satellite data by variance components, J. Geodesy, 76, 259-268, doi:10.1007/s00190-002-0245-x, 2002.

Kourisa, S. S., Polimerisa, K. V., Canderb, L. R., and Ciraoloc, L.: Solar and latitude dependence of TEC and SLAB thickness, J. Atmos. Sol.-Terr. Phys., 70, 1351-1365, doi:10.1016/j.jastp.2008.03.009, 2008.

Liang, W., Limberger, M., Schmidt, M., Dettmering, D., and Hugentobler, U.: Combination of ground- and space-based GPS data for the determination of a multi-scale regional 4-D ionosphere model, International Association of Geodesy Symposia, doi:10.1016/j.asr.2014.12.006, accepted, 2014.

Limberger, M., Liang, W., Schmidt, M., Dettmering, D., and Hugentobler, U.: Regional representation of F2 Chapman parameters based on electron density profiles, Ann. Geophys., 31, 2215-2227, doi:10.5194/angeo-31-2215-2013, 2013.

Liu, L., Wan, W., and Ning, B.: A study of the ionogram derived effective scale height around the ionospheric hmF2, Ann. Geophys., 24, 851-860, doi:10.5194/angeo-24-851-2006, 2006.

Lee, C.-C. and Reinisch, B. W.: Quiet-condition variations in the scale height at F2-layer peak at Jicamarca during solar minimum and maximum, Ann. Geophys., 25, 2541-2550, doi:10.5194/angeo-25-2541-2007, 2007.
Lyche, T. and Schumaker, L. L.: A multiresolution tensor spline method for fitting functions on the sphere, SIAM J. Sci. Comput., 22, 724-746, doi:10.1137/S1064827598344388, 2000.

Nava, B., Coïsson, P., and Radicella, S. M.: A new version of the NeQuick ionosphere electron density model, J. Atmos. Sol.-Terr. Phys., 70, 1856-1862, 2008.

Reinisch, B. W., Huang, X.-Q., Belehaki, A., Shi, J.-K., Zhang, M.-L., and Ilma, R.: Modeling the IRI topside profile using scale heights from ground-based ionosonde measurements, Adv. Space Res., 34, 2026-2031, doi:10.1016/j.asr.2004.06.012, 2004.

Rocken, C., Kuo, Y.-H., Schreiner, W. S., Hunt, D., Sokolovskiy, S., and McCormick, C.: COSMIC System Description, Terr. Atmos. Ocean. Sci., 11, 21-52, 2000.

Sánchez, L., Seemüller, W., Drewes, H., Mateo, L., González, G., Silva, A., Pampillón, J., Martínez, W., Cioce, V., Cisneros, D., and Cimbaro, S.: Long-Term Stability of the SIRGAS Reference Frame and Episodic Station Movements Caused by the Seismic Activity in the SIRGAS Region, IAG Symposia, 138, 153-161, Springer Berlin Heidelberg, 2013.

Schmidt, M.: Wavelet modeling in support of IRI, Adv. Space Res., 39, 932-940, doi:10.1016/j.asr.2006.09.030, 2007.

Schmidt, M., Dettmering, D., Mößmer, M., Wang, Y., and Zhang, J.: Comparison of spherical harmonic and B-spline models for vertical total electron content, Radio Sci., 46, RS0D11, doi:10.1029/2010RS004609, 2011.

Schunk, R. W., Scherliess, L., Sojka, J. J., Thompson, D. C., Anderson, D. N., Codrescu, M., Minter, C., Fuller-Rowell, T. J., Heelis, R. A., Hairston, M., Howe B.: Global Assimilation of Ionospheric Measurements (GAIM), Radio Sci., 39, RS1S02, doi:10.1029/2002RS002794, 2001.

Stankov, S. M. and Jakowski, N.: Topside plasma scale height modelling based on CHAMP measurements: first results, Earth Observation with CHAMP - Results from Three Years in Orbit, Springer book, XVI, Springer, ISBN 3-540-22804-7, 2005.

Todorova, S., Schuh, H., Hobiger, T., and Hernández-Parajes, M.: Global ionosphere models obtained by integration of GNSS and satellite altimetry data, Österreichische Zeitschrift für Vermessung und Geoinformation (VGI), 2, 80-89, 2007.

Tsai, L.-C., Tsai, W.-H. Schreiner, W. S., Berkey, F. T., and Liu, J. Y.: Comparisons of GPS/MET retrieved ionospheric electron density and ground based ionosonde data, Earth Planet. Space, 53, 193-205, 2001.

Tsai, L.-C. and Tsai, W.-H.: Improvement of GPS/MET ionospheric profiling and validation using the Chung-Li ionosonde measurements and the IRI model: Terrestrial, Atmos. Ocean. Sci., 15, 589-607, doi:10.3319/TAO.2007.12.19.01(F3C), 2004.

Tsai, L.-C., Liu, C. H., and Hsiao, T. Y.: Profiling of ionospheric electron density based on FormoSat-3/COSMIC data: results from the intense observation period experiment: Terrestrial, Atmos. Ocean. Sci., 20, 181-191, 2009.

Wright, J. W.: A model of the F region above $h m \mathrm{~F} 2$, J. Geophys. Res., 65, 185-191, 1960.

Zhang, M.-L., Reinisch, B. W., Shi, J.-K., Wu, S.-Z., and Wang, $\mathrm{X}$.: Diurnal and seasonal variation of the ionogram-derived scale height at the F2 peak, Adv. Space Res., 37, 967-971, doi:10.1016/j.asr.2006.02.0042, 2006. 